SAMMLUNG TUSCULUM

Wissenschaftliche Beratung:

Gerhard Fink, Niklas Holzberg,

Rainer Nickel, Bernhard Zimmermann 



\title{
CORNELIUS NEPOS
}

\section{BERÜHMTE MÄNNER}

\section{DE VIRIS ILLUSTRIBUS}

\author{
Lateinisch-deutsch
}

Herausgegeben und übersetzt von Michaela Pfeiffer unter Mitarbeit von Rainer Nickel 


\section{Für Lukas und Sebastian}

Bibliographische Information der Deutschen Bibliothek

Die Deutsche Bibliothek verzeichnet diese Publikation in der Deutschen Nationalbibliographie; detaillierte bibliographische Daten sind im Internet unter http://dnb.ddb.de abrufbar.

(C) 2006 Patmos Verlag GmbH \& Co. KG

Artemis \& Winkler Verlag, Düsseldorf

Alle Rechte vorbehalten.

Druck und Verarbeitung: Friedrich Pustet, Regensburg

ISBN 3-7608-I734-3

www.patmos.de 